\title{
Knockdown of ribosomal protein S15A induces human glioblastoma cell apoptosis
}

Chen Zhang ${ }^{1 \dagger}$, Jiqiang Fu ${ }^{1 \dagger}$, Fei Xue ${ }^{1 \dagger}$, Bomi Ryu ${ }^{2}$, Ting Zhang ${ }^{3}$, Shuili Zhang ${ }^{4}$, Jingyu Sun ${ }^{5}$, Xinxin Xu', Zhaoli Shen ${ }^{1}$, Longpo Zheng ${ }^{1 *}$ and Xianzhen Chen ${ }^{1 *}$

\begin{abstract}
Background: RPS15A is a ribosome protein that is highly conserved in many organisms from yeast to human. A number of studies implied its role in promoting cancer cell growth.

Methods: Here, we firstly conducted RPS15A gene expression analysis in brain cancer using Oncomine database and found RPS15A was remarkably overexpressed in glioblastoma (GBM) compared with that in normal tissues. Then, the expression of RPS15A was specifically silenced in GBM cell line U251 using lentiviral-mediated RNA interference technique. We further investigated the effect of RPS15A knockdown in U251 cells using MTT assay, colony formation test, and flow cytometry analysis. We detected the protein level of Bcl-2 and poly (ADP-ribose) polymerase (PARP) as well as activation of caspase-3.

Results: Our results showed that the knockdown of RPS15A could inhibit cancer cell growth and colony formation in vitro, as well as induced cell cycle arrest at G0/G1 phase and cell apoptosis. In addition, Western blot analysis indicated that the knockdown of RPS15A could significantly inhibit bcl-2 and activate caspase-3 and PARP.

Conclusions: Our findings suggest RPS15A may play an important role in the progression of GBM and lentiviralmediated silencing of RPS15A could be an effective tool in GBM treatment.
\end{abstract}

Keywords: Glioblastoma, RPS15A, Cell proliferation, Apoptosis

\section{Background}

Glioma is the most common and most aggressive malignant brain tumor in the human central nervous system. According to histological progression, gliomas are classified into four grades, including low-grade astrocytomas (grade I-II), anaplastic astrocytomas (grade III), and glioblastoma (GBM, grade IV) [1, 2]. As a frequent form of malignant glioma [3], GBM is invariably lethal for patients at most 2 years following diagnosis [4]. Current standard treatment for patients with GBM is concurrent TMZ and radiotherapy followed by six maintenance cycles of adjuvant chemotherapy to improve progressionfree survival and overall survival [5]. Despite the standard of care therapy, recurrence rates remain higher in patients with GBM. Therefore, it is urgent to

\footnotetext{
* Correspondence: zhenglongpoy@163.com; chenxianzheny@126.com ${ }^{\dagger}$ Equal contributors

'Shanghai Tenth People's Hospital, Tongji University School of Medicine, 301 Yanchang Road, Shanghai 200072, China

Full list of author information is available at the end of the article
}

identify new molecular targets to improve the prognosis against human GBM.

Ribosome is the fundamental translational machinery for protein synthesis from messenger RNAs. In eukaryotes, a mature ribosome consists of $60 \mathrm{~S}$ and $40 \mathrm{~S}$ subunits [6]. Ribosome biogenesis and protein translation are fine-tuned to match the cell growth rate, proliferation, and differentiation during development. However, this type of regulation is abnormal in cancer cells [7]. Several tumor suppressors could inhibit tumor cell growth by affecting ribosome biogenesis [8]. On the other hand, oncogenes often stimulate cell growth and proliferation by enhancing ribosome biogenesis in cancer cells [9]. RPS15A, ribosomal protein s15a, is one of the subunits of the $40 \mathrm{~S}$ ribosomal protein $[10,11]$. To data, there are several preliminary studies which show that down-regulation of RPS15A suppresses proliferation of lung cancer cells, osteosarcoma cells, and hepatic cancer cells in vitro [12-14]. Despite the ubiquitous presence and important functions of ribosomal complex, the role 
of ribosomal protein RPS15A in GBM cells remains not to be fully understood.

Herein, we aimed to investigate the effect of RPS15A knockdown in GBM progression. We firstly found the messenger RNA (mRNA) levels of RPS15A were significantly up-regulated in glioma compared that in normal tissues using the datasets from Oncomine database. Functional analysis showed knockdown of RPS15A obviously suppressed cell proliferation in U251 by inducing cell cycle arrest and apoptosis. More interestingly, we found RPS15A knockdown induced apoptosis through cell intrinsic mitochondria pathway. Our results suggested a prominent role of RPS15A in GBM cell proliferation and provide a new insight to a potential therapeutic target for GBM.

\section{Methods}

\section{Cell lines and culture}

The human GBM cell line U251 and the human embryonic kidney cell line 293T (HEK293T) were purchased from Cell Bank of Chinese Academy of Science (Shanghai). Cells were cultured in DMEM (Hyclone) supplemented with $10 \% \mathrm{FBS}$ (Biological Industries) at $37{ }^{\circ} \mathrm{C}$ in an incubator containing $5 \% \mathrm{CO}_{2}$.

\section{Construction of RPS15A short hairpin RNA containing lentivirus}

For the RNA interfering experiment, two short hairpin RNA (shRNA) sequences were designed against the RPS15A gene (NM_001019): shRPS15A-1, 5'-GTGC AACTCAAAGACCTGGAACTCGAGTTCCAGGTCTT 'TGAGTTGCACTTTTT-3' and shRPS15A-2, 5'-GCA TGGTTACATT-GGCGAATTCTCGAGAATTCGCCA ATGTAACCATGCTTTTT-3'). The control shRNA (shCon) sequence was $5^{\prime}$-GCGGAGGGTTTGAAAGAA TATCTCGAGATATTCTTTCAAACCCTCCGCTTTTT T-3'. Each nucleotide sequence was synthesized and inserted into $\mathrm{pFH}-\mathrm{L}$ shRNA expressing vector. For lentiviral production, recombinant plasmid described above and pHelper plasmids (pVSVG-1 and pCMVD8.92) were co-transfected into HEK293T cells using Lipofectamine 2000 (Invitrogen) according to the manufacturer's instruction. After $72 \mathrm{~h}$, virus supernatant was harvested and concentrated by ultracentrifugation $\left(4000 g\right.$ at $\left.4{ }^{\circ} \mathrm{C}\right)$ for $10 \mathrm{~min}$.

\section{Cell infection}

For cell infection, U251 cells were seeded at a density of 50,000 cells per well in six-well plates and transduced with constructed lentiviruses (shCon, shRPS15A-1, and shRPS15A-2) at a multiplicity of infection of 40. After $72-\mathrm{h}$ infection, the infection efficiency was determined by observing the green fluorescence protein (GFP) expression under a fluorescence microscope.

\section{Quantitative PCR analysis}

After 6-day infection, total RNA was isolated from cells by using the Trizol reagent (Invitrogen), according to the manufacturer's protocol. Reverse transcribed was performed Bio-Rad Connect Real-Time PCR (polymerase chain reaction) platform (Bio-Rad Laboratories Inc, Hercules, CA, USA) using an SYBR Green Master Mix Kit. Data were analyzed using the $2^{\Delta \Delta C t}$ method, and the levels of mRNA were normalized to $\beta$-actin. The PCR primers used were as follows: RPS15A (forwardCCTCCTTTTTCGGTTTCCTC; reverse-AGAGATGG AA-TGGTGGTTGG); $\beta$-actin (forward-GTGGACATCC GCAAAGAC; reverse-AAAGGGTGT-AACGCAACTA).

\section{Western blot analysis}

Western blot analysis was done 5 days post infection. Proteins were extracted from cells using $2 \times$ SDS sample buffer (100 mM Tris- $\mathrm{HCl}$ (pH 6.8), 10 mM EDTAm $4 \%$ SDS, 10 Glycine. A total of 30 ug proteins were separated in $12 \%$ SDS-PAGE and transferred to PVDF membranes. The membranes were incubated with primary antibodies (rabbit anti-RPS15A, 1:1000, Ab175054, Abcam; rabbit anti-caspase-3, 1:500, \#9661, Cell signaling; rabbit anti-poly (ADP-ribose) polymerase (PARP), 1:1000, \#9542, Cell signaling; rabbit anti-bcl-2, 1:1000, \#2876, Cell signaling; rabbit anti-GAPDH, 1:500000, \#2876, Cell signaling) for $1 \mathrm{~h}$ at room temperature followed by incubation with secondary antibody goat anti-rabbit HRP (Santa Cruz) at room temperature for $1 \mathrm{~h}$. The bands were visualized using an ECL kit (Beyotime). Protein bands were quantified using Gel-pro analyzer software (MediaCybernetics). GAPDH was used as the reference control.

\section{MTT assay}

Cells were seeded in a 96-well plate with 2500 cells per well. Growth curve and MTT assay was carried out $96 \mathrm{~h}$ post transduction of virus. Briefly, MTT solution was added to each well, followed by $4 \mathrm{~h}$ of incubation at $37^{\circ} \mathrm{C}$. Then, cells were washed and dissolved in acidic isopropanol (10 \% SDS, $5 \%$ isopropanol, and $0.01 \mathrm{~mol} / \mathrm{L} \mathrm{HCl}$ ) for $10 \mathrm{~min}$. Cell density was measured at $595 \mathrm{~nm}$ using the microplate reader using enzyme-linked immunosorbent assay. The cell growth curves were drawn according to the OD values.

\section{Colony formation analysis}

Cells were seeded at the density of 500 cells per well in a six-well plate. After $96 \mathrm{~h}$ infection with shRNA virus, followed by additional eight days of incubation, cells were washed with PBS twice, fixed with absolute methanol for $15 \mathrm{~min}$. Then, fixed cells were stained with $1 \%$ crystal violet for $20 \mathrm{~min}$. After cleaning with PBS, colonies were counted under light microscope. 


\section{Cell cycle and apoptosis analysis}

Cells were seeded in a 6 -cm dish at 100,000 cells per well. Four days after infection with lentivirus, the cells were fixed with pre-cooled $70 \%$ ethanol at $4{ }^{\circ} \mathrm{C}$ overnight and incubated with $1 \mathrm{mg} / \mathrm{ml}$ RNase A (QIAGEN) for $30 \mathrm{~min}$ at $37{ }^{\circ} \mathrm{C}$. Then, cells were added propidium iodide (50 ug/mL, ebioscience) at $4{ }^{\circ} \mathrm{C}$ for $30 \mathrm{~min}$ to stain DNA. The DNA content of cells was determined by a FACS Calibur II sorter and Cell Quest FACS system (BD Biosciences).

For cell apoptosis analysis, cells were harvested after 3-day infection with lentivirus and resuspended in 100 ul $1 \times$ binding buffer (ebioscience). Then, cells were stained with $2 \mathrm{uL}$ Annexin V-APC $(20 \mathrm{ug} / \mathrm{ml}$; ebioscience) for $15 \mathrm{~min}$ on ice. Samples were diluted to $400 \mathrm{uL}$ and added $1 \mathrm{uL} 7-\mathrm{AAD}$ (50 ug/ml; ebioscience) before detection on FACS Calibur II sorter and Cell Quest FACS system (BD Biosciences).

\section{Statistical analysis}

All experiments were at least repeated in triplicate. All data were analyzed using GraphPad Prism software and expressed as the mean \pm standard deviation (SD) of three independent experiments. Comparisons between groups were conducted by Student's $t$ test. The $p$ value $<0.05$ was considered as statistically significant.

\section{Results}

\section{RPS15A is overexpressed in GBM}

In order to evaluate the differential expression of RPS15A in GBM, we used the Oncomine database (www.oncomine.org), a cancer microarray database of genome-wide expression analysis [15]. A quick search of RPS15A gene in GBM revealed that this gene was upregulated in several types of GBM, including anaplastic oligoastrocytoma [16, 17], glioblastoma [16, 18], oligodendroglioma [19], and diffuse astrocytoma [20] vs. normal tissues (Table 1). The comparison showed an average increase of one- to sixfold in RPS15A expression in GBM compared to normal tissues. Therefore, we concluded that RPS15A might play an important role in GBM progression. Thus, we focused on this particular gene in following studies by using U251, a cell line derived from a malignant glioblastoma.

\section{RPS15A gene knockdown in U251 cells}

To investigate the biological function of RPS15A in glioblastoma cells, we constructed two individual shRNA vectors for RPS15A knockdown using lentiviral vectors carrying green fluorescent protein (GFP). Both control vectors (shCon) and two target vectors (shRPS15A-1 and shRPS15A-2) showed good infection rates $72 \mathrm{~h}$ post infection in U251 cells, determined by a fluorescence microscopy of the GFP expression (Fig. 1a, b). We performed RT-PCR and western blot to evaluate the efficiency of shRNA knockdown of RPS15A. As shown in Fig. 1c, d, both of two target shRNAs showed significant reduction of RPS15A mRNA compared to the shCon $(p<0.001)$. Western blot confirmed that, at protein level, target shRNAs efficiently knocked down RPS15A expression in U251 cells (Fig. 1e). Together, these results suggested that we successfully constructed RPS15A knockdown cell model, which could be applied to the following studies.

\section{RPS15A knockdown affects cell proliferation and colony formation ability}

We next examined the effect of RPS15A on cell proliferation and viability. We measured cell proliferation by MTT assay, a colorimetric assay assessing cell metabolic activity depending on NADPH oxidoreductase enzymes, over the course of 5 days. As shown in Fig. 2a, shRPS15A-treated cells showed significant inhibition of cell proliferation compared to cells treated with shCon $(p<0.001)$. We also did colony formation assay with cells treated by shCon vs. shRPS15A. As shown in Fig. 2b, c, shRPS15A-treated cells failed to form any colony, whereas shCon-treated cells can form colonies

Table 1 RPS15A differential transcript expression in human glioblastoma extracted from multiple studies in the Oncomine database

\begin{tabular}{llcl}
\hline Study & Comparison (specimen number in each group) & Fold change & $p$ value \\
\hline Bredel M et al. & Anaplastic oligoastrocytoma (6) vs. normal(4) & 2.125 & 0.005 \\
Bredel M et al. & Anaplastic oligodendroglioma (3) vs. normal (4) & 2.337 & 1.916 \\
Bredel M et al. & Glioblastoma (5) vs. normal (4) & 2.014 & 0.004 \\
Bredel M et al. & Oligodendroglioma (27) vs. normal (4) & 2.373 & $0.64 \mathrm{E}-04$ \\
French PJ et al. & Anaplastic oligodendroglioma (23) vs. normal(6) & 1.985 & $4.006 \mathrm{E}-05$ \\
Murat A et al. & Glioblastoma (80) vs. normal(4) & 5.803 & $1.51 \mathrm{E}-08$ \\
Shai R et al. & Oligodendroglioma (3) vs. normal (7) & 2.163 & $5.49 \mathrm{E}-06$ \\
Sun L et al. & Diffuse astrocytoma (7) vs. normal (23) & 1.408 & $8.40 \mathrm{E}-07$ \\
TCGA & Brain glioblastoma (542) vs. normal (10) & $7.04 \mathrm{E}-04$ \\
\hline
\end{tabular}

TCGA The Cancer Genome Atlas-Glioblastoma Multiforme Gene Expression Data (http://tcga-data.nci.nih.gov/tcga/) 


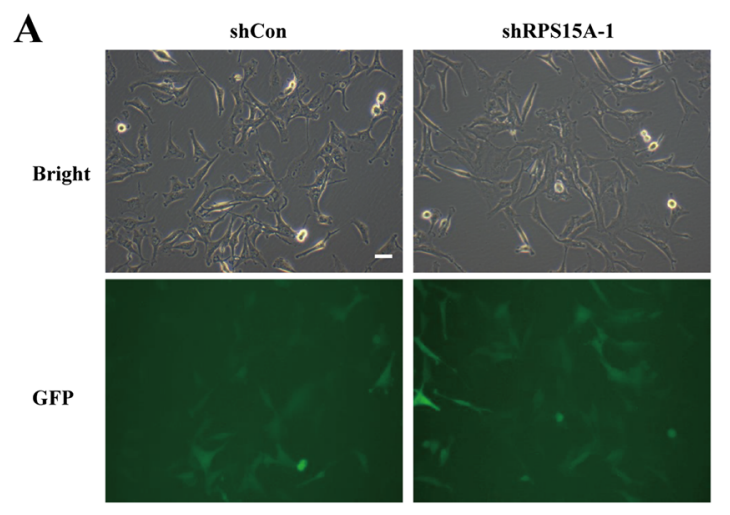

B

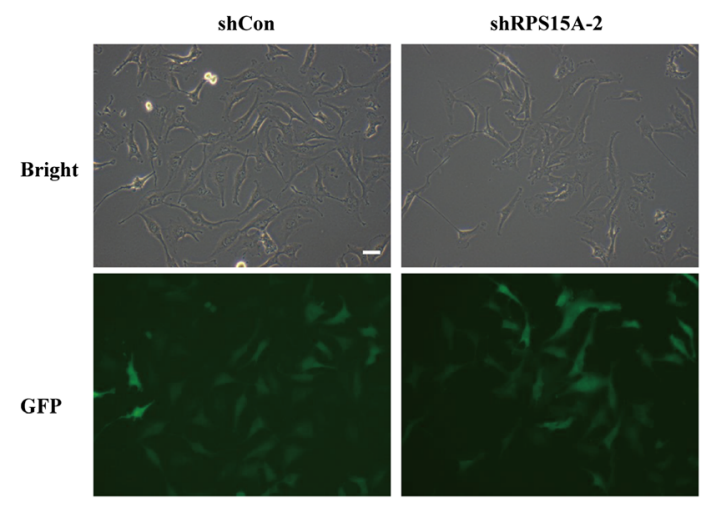

C

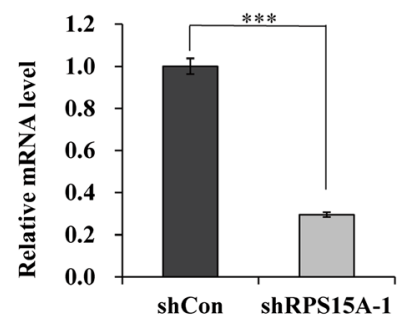

D

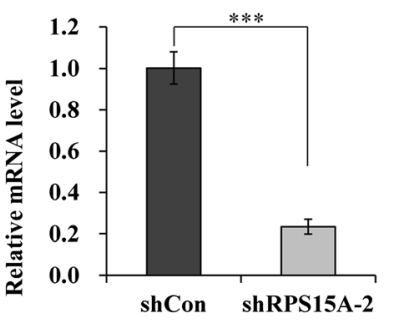

$\mathbf{E}$

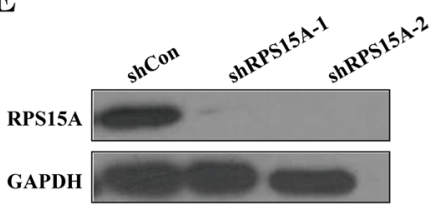

Fig. 1 Verification of RPS15A silencing in U251 cells after shRPS15A infection. a, b Infection efficiency was assessed by counting GFP-positive cells with fluorescence microscopy. $\mathbf{c}$, d The mRNA levels of RPS15A in U251 cells with three treatments (shCon, shRPS15A-1, and shRPS15A-2) measured by qPCR analysis. The experiment was performed in triplicate and repeated three times. e The protein levels of RPS15A in U251 cells with three treatments (shCon, shRPS15A-1, and shRPS15A-2) determined by Western blot analysis. ${ }^{* *} p<0.001$

naturally $(p<0.01)$. Therefore, RPS15A could promote cell viability and proliferation ability.

RPS15A plays a role in cell cycle progression in U251 cells Inhibition of cell proliferation could occur at any stage of the cell cycle. To investigate the mechanisms of the U251 cell proliferation inhibition induced by shRPS15A, we used flow cytometry to examine the cell cycle progression under the treatment of shCon or shRPS15A (Fig. 3a). We found that $72 \mathrm{~h}$ after shRNA treatment, shRPS15Atreated cells showed significantly reduced cells in $\mathrm{S}$ phase compared to shCon-treated cells $(p<0.001)$; instead, there were more cells accumulated in G0/G1 phase $(p<0.001)$ after shRPS15A infection, but no significant difference was found in G2/M phase (Fig. 3b). This cell cycle results strongly indicated that knockdown of RPS15A arrested cell cycle at G0/G1 phase, which means RPS15A plays an important role in cell cycle progress from $\mathrm{G} 1$ to $\mathrm{S}$ phase.

\section{RPS15A knockdown induces apoptosis in U251 cells}

To further investigate the effect of RPS15A on cell apoptosis, Annexin V combined with 7-Aminoactinomycin D (7-AAD) staining was used to distinguish apoptosis
(Fig. 4a) between early and late stages of apoptosis based on the exposure of phosphatidylserine to the surface of cell apoptosis [21]. We found that knockdown of RPS15A significantly induced cell early (Annexin $\mathrm{V}+/ 7-$ $\mathrm{AAD}-$ ) and late apoptosis (Annexin $\mathrm{V}+/ 7-\mathrm{AAD}+$ ) in U251 cells (Fig. 4b, $p<0.001$ ).

We further extended our study to investigate the pathway through which RPS15A knockdown induced apoptosis. Apoptosis is typically induced through two distinct signaling pathways: the death receptor pathway and mitochondrial pathway [22]. Because RPS15A is a cytosolic protein, we hypothesized it affected mitochondria pathway, in which bcl-2, caspase 3, and PARP are critical components. Thus, we carried out Western blot analysis to determine the expression of these three proteins in shRPS15A-treated cells. As shown in Fig. 4c, the antiapoptotic and mitochondria localized protein Bcl-2 was down-regulated in U251 cells after RPS15A knockdown. The expression levels of cleaved caspase- 3 and PARP were up-regulated in U251 cells following shRPS15A infection. This result indicated that RPS15A knockdown activated the mitochondrial pathway of apoptosis. What is more, shRPS15A-1 showed more strong effect on 


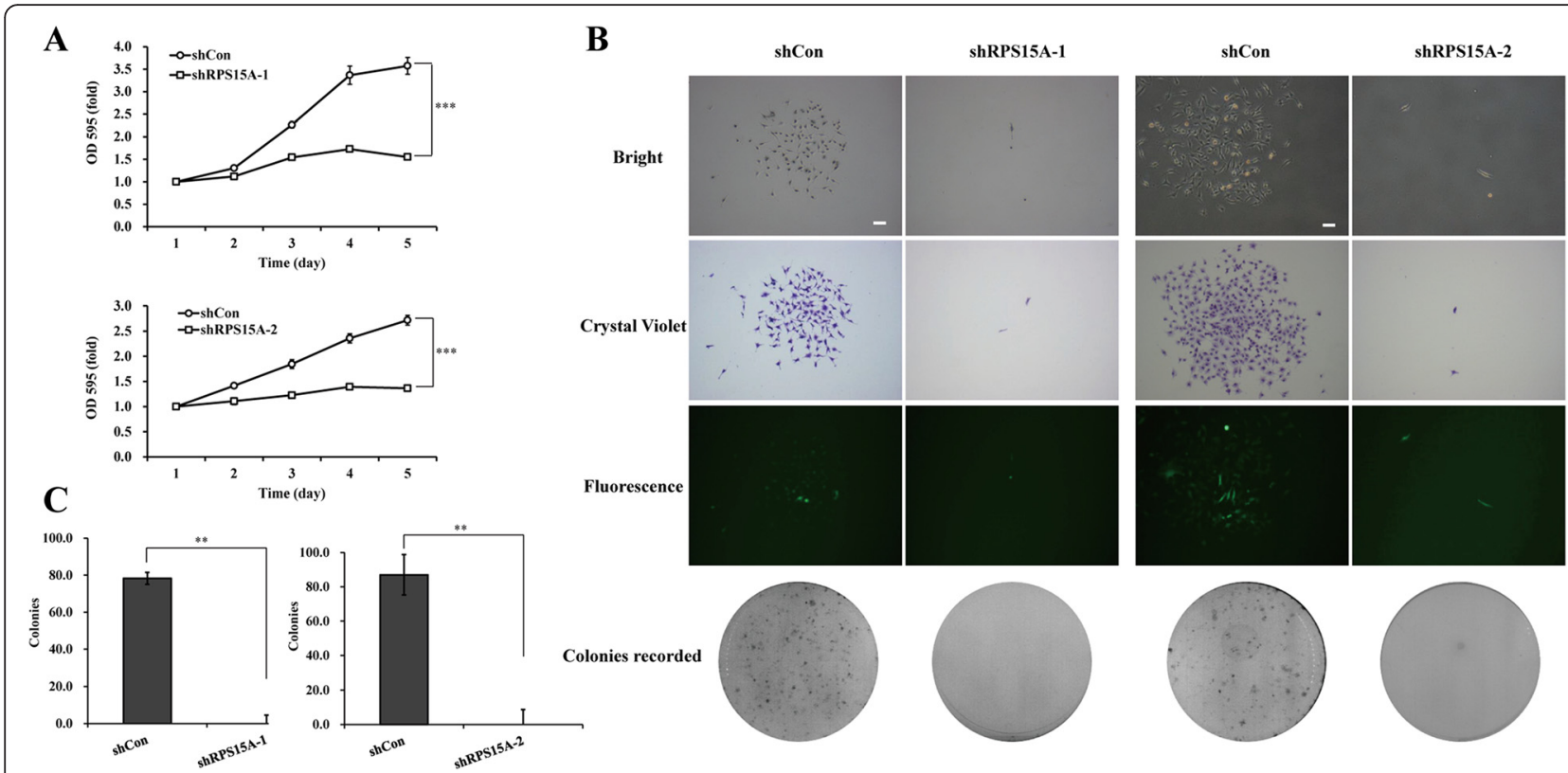

Fig. 2 Lentivirus-mediated knockdown of RPS15A suppresses cell viability and the colony formation ability of U251 cells. a Growth curve of U251 cells with three treatments (shCon, shRPS15A-1, and shRPS15A-2) determined by MTT assay. b Representative images of colonies formed in U251 cells with three treatments (shCon, shRPS15A-1, and shRPS15A-2) measured by the colony formation assay. c Statistical analysis of colony formation ability as shown. The experiments was performed in triplicate and repeated three times. ${ }^{* *} p<0.01,{ }^{* * *} p<0.001$

apoptosis induction than shRPS15A-2. Taken together, we found that RPS15A knockdown induced U251 cells apoptosis through mitochondrial pathway.

\section{Discussion}

RPS15A encodes a 40S ribosomal protein S15a, one of the components of the 40S subunit in human [23]. Ribosomes are required for basic cellular functions, especially for cancer cells, whose growth and proliferation are uncontrolled, thus demand a large amount of protein synthesis [7]. GBM is invariably lethal glioma characterized by extremely high aggressiveness. However, little is known about the biological function of ribosome formation in GBM progression. In this study, we firstly evaluated the relative expression of RPS15A in glioma tissues and normal tissues and found it was significantly upregulated in GBM tissues compared with that in normal tissues. To further investigate its role in GBM, the functional role of RPS15A in human GBM cell growth was studied by RNAi-mediated knockdown. Our results

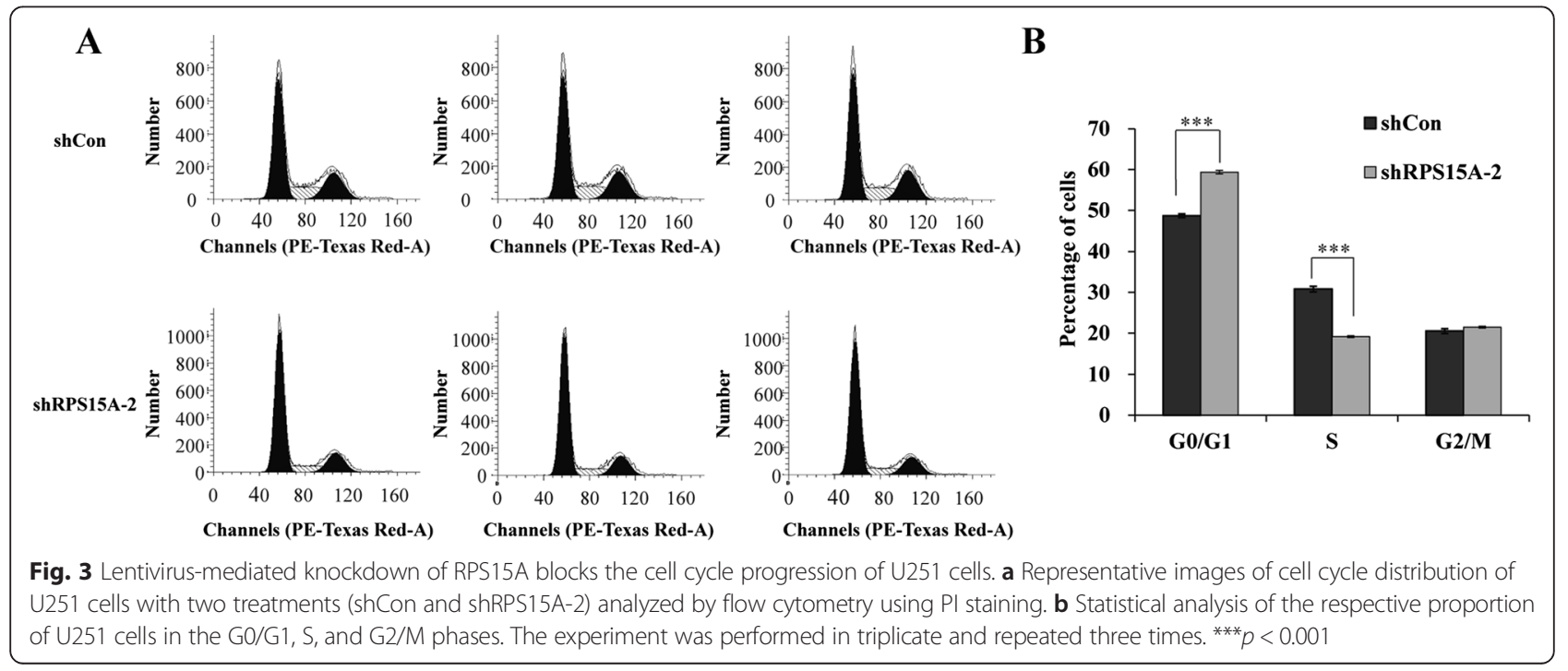



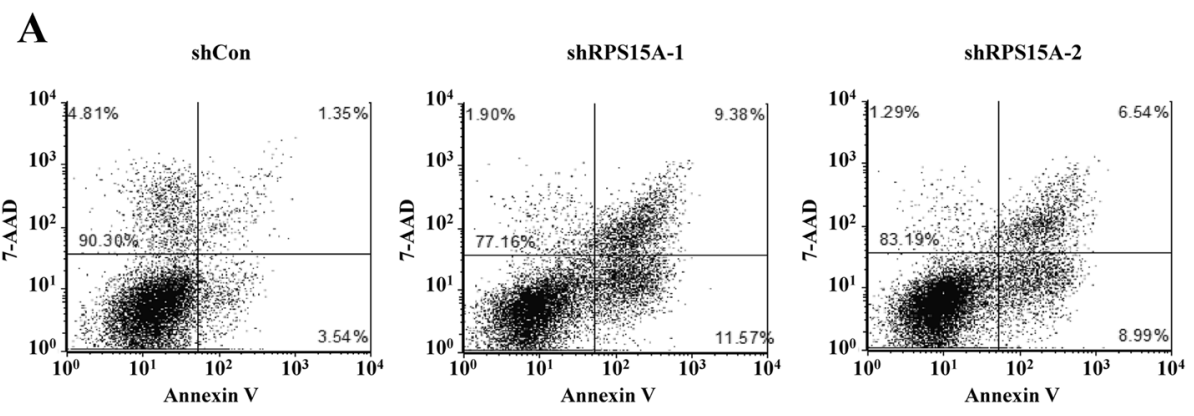

\section{B}

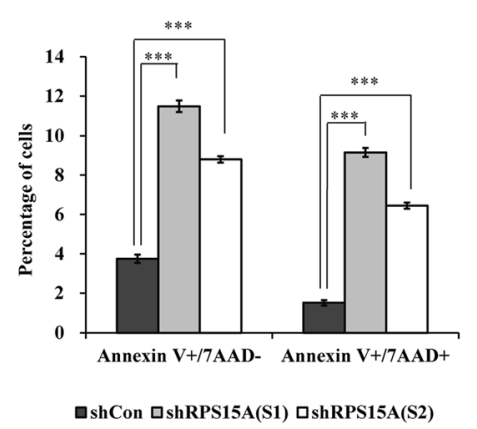

C

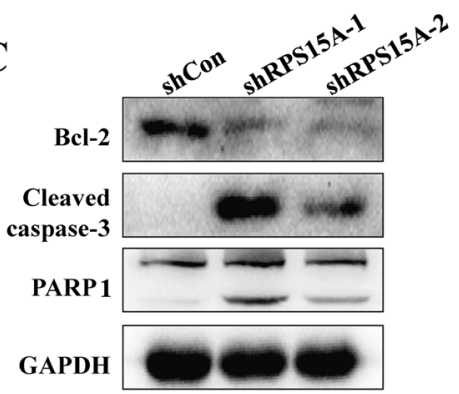

Fig. 4 Knockdown of RPS15A induces cell apoptosis via activation of mitochondrial pathway. a Representative images of cell apoptosis of U251 cells with three treatments (shCon, shRPS15A-1, and shRPS15A-2) analyzed by flow cytometry using Annexin V/7-AAD double staining. b Statistical analysis of the proportions of $\mathrm{U} 251$ cells corresponding to early (Annexin V+/7-AAD-) and late (Annexin V+/7-AAD+) apoptotic cells. c Activation of bcl-2, caspase-3, and PARP signaling pathways measured by Western blot analysis. The experiment was performed in triplicate and repeated three times. ${ }^{* * *} p<0.001$

showed knockdown of RPS15A remarkably suppressed U251 cell growth, possibly due to induction of cell cycle arrest and apoptosis.

Similar effects of RPS15A were demonstrated in previous studies in other cancer types [13, 24]. Our study demonstrated that RPS15A was important for survival and proliferation of glioblastoma cancer cells. In the absence of this gene, the cells underwent apoptosis through intrinsic mitochondria pathway.

The mitochondrial pathway of apoptosis typically involves the release of mitochondrial cytochrome $\mathrm{c}$ into cytosol. The released cytochrome c combines with apoptotic protease activating factor 1 (Apaf-1) and procaspase- 9 , resulting in the activation of caspse- 9 and caspase-3 [25]. Bcl-2 plays an important role in releasing cytochrome c from mitochondria. Our western blot result of Bcl-2 showed a significant decrease upon RPS15A knockdown. Thus, wastage of RPS15A led to the increase of anti-apoptotic signals (Bcl-2), which was in favor of the occurrence of apoptosis. Caspase-3 cleavage and PARP cleavage are the downstream of $\mathrm{Bcl}-2$ [26, 27]. Our western blot confirmed these two cleavage events were both enhanced upon RPS15A knockdown. These results suggested that RPS15A knockdown induced apoptosis mainly through the activation of mitochondrial pathway. We hypothesize that RPS15A might be directly involved in the translation of Bcl-2 mRNA; thus, knockdown of RPS15A resulted in the down-regulation of Bcl2 protein.

Many types of cancers, including glioblastoma, are resistant against many anti-cancer treatments. One of the main reasons is that they do not activate their intracellular apoptosis pathway [28]. Current standard chemotherapy treatment for glioblastoma is the use of alkylating agents, temozolomide, in combination with radiation therapy. Temozolomide has been proven somewhat effective in prolonging survival; however, tumor often returns after treatment, which begs the exploration of new drugs and therapies. Our finding of induction of apoptosis in GBM cell line by down-regulating RPS15A provides a new avenue, which targets ribosome proteins.

\section{Conclusions}

From above analysis, we can see that RPS15A was significantly up-regulated in GBM tissues compared with that in normal tissues. Knockdown of RPS15A remarkably suppressed U251 cell growth; then, the results demonstrated that RPS15A was important for survival and proliferation of glioblastoma cancer cells. In the absence of this gene, the cells underwent apoptosis through activation of intrinsic mitochondria pathway. 
In summary, our findings suggest RPS15A may play an important role in the progression of GBM and lentiviralmediated silencing of RPS15A could be an effective tool in GBM treatment.

\section{Competing interests}

The authors declare that they have no competing interests.

\section{Authors' contributions}

CZ carried out the construction of RPS15A short hairpin RNA containing lentivirus, participated in the cell cycle and apoptosis analysis, and drafted the manuscript. JF carried out the cell infection and quantitative PCR analysis. FX participated in the cell cycle and apoptosis analysis. TZ participated in the cell culture. SZ participated in the Western blot analysis. JS participated in the MTT assay. ZS participated in the colony formation analysis. LZ participated in the design of the study and performed the statistical analysis. XC conceived of the study and participated in its design and coordination and helped to draft the manuscript. BR and XX participated in the grammar mistakes correcting and statistical analysis. All authors read and approved the final manuscript.

\section{Acknowledgements}

The authors are thankful for the financial support from the Shanghai Municipal Commission of Health and Family Planning No. 20154Y0038, project 81550110259 supported by the National Natural Science Foundation of China Science, Technology Innovation Foundation of Planted Talent Plan for Undergraduate in Zhejiang Province No. 2015R410009, and Natural Science Foundation of Zhejiang Province No. Y12H280030.

\section{Author details}

'Shanghai Tenth People's Hospital, Tongji University School of Medicine, 301 Yanchang Road, Shanghai 200072, China. ${ }^{2}$ Shanghai Municipal Hospital of Traditional Chinese Medicine, Shanghai University of Traditional Chinese Medicine, 274 Middle Zhijiang Road, Shanghai 200071, China. ${ }^{3}$ College of Medical Technology, Zhejiang Chinese Medical University, Hangzhou 310053, China. ${ }^{4}$ College of Pharmacy, Zhejiang Chinese Medical University, Hangzhou 310053, China. ${ }^{5}$ Sports and Health Research Center, Tongji University Department of Physical Education, Shanghai 200092, China.

Received: 28 December 2015 Accepted: 21 April 2016

\section{Published online: 29 April 2016}

\section{References}

1. Kleihues P, Burger PC, Scheithauer BW. The new WHO classification of brain tumours. Brain Pathol. 1993;3(3):255-68.

2. Kleihues P, et al. The WHO classification of tumors of the nervous system. J Neuropathol Exp Neurol. 2002;61(3):215-25. discussion 226-9.

3. Kang TW, et al. Growth arrest and forced differentiation of human primary glioblastoma multiforme by a novel small molecule. Sci Rep. 2014;4:5546.

4. Jensen $\mathrm{SA}$, et al. Spherical nucleic acid nanoparticle conjugates as an RNAibased therapy for glioblastoma. Sci Transl Med. 2013;5(209):2124-34.

5. Stupp R, et al. Radiotherapy plus concomitant and adjuvant temozolomide for glioblastoma. N Engl J Med. 2005;352(10):987-96.

6. Fatica A, Tollervey D. Making ribosomes. Curr Opin Cell Biol. 2002;14(3):313-8.

7. Ruggero D, Pandolfi PP. Does the ribosome translate cancer? Nat Rev Cancer. 2003;3(3):179-92.

8. Ciarmatori $\mathrm{S}$, et al. Overlapping functions of the $\mathrm{pRb}$ family in the regulation of rRNA synthesis. Mol Cell Biol. 2001;21(17):5806-14.

9. Barna M, et al. Suppression of Myc oncogenic activity by ribosomal protein haploinsufficiency. Nature. 2008;456(7224):971-5.

10. Wool IG, Chan YL, Gluck A. Structure and evolution of mammalian ribosomal proteins. Biochem Cell Biol. 1995;73(11-12):933-47.

11. Chan YL, et al. The primary structure of rat ribosomal protein S15a. Biochem Biophys Res Commun. 1994;200(3):1498-504.

12. Zhao X, et al. Decreased expression of RPS15A suppresses proliferation of lung cancer cells. Tumour Biol. 2015;36(9):6733-40.

13. Zhang C, et al. Ribosomal protein S15A augments human osteosarcoma cell proliferation in vitro. Cancer Biother Radiopharm. 2014;29(10):451-6.

14. Xu M, et al. Down-regulation of ribosomal protein S15A mRNA with a short hairpin RNA inhibits human hepatic cancer cell growth in vitro. Gene. 2014; 536(1):84-9.
15. Rhodes DR, et al. ONCOMINE: a cancer microarray database and integrated data-mining platform. Neoplasia. 2004;6(1):1-6.

16. Bredel $\mathrm{M}$, et al. Functional network analysis reveals extended gliomagenesis pathway maps and three novel MYC-interacting genes in human gliomas. Cancer Res. 2005;65(19):8679-89.

17. French PJ, et al. Gene expression profiles associated with treatment response in oligodendrogliomas. Cancer Res. 2005;65(24):11335-44.

18. Murat A, et al. Stem cell-related "self-renewal" signature and high epidermal growth factor receptor expression associated with resistance to concomitant chemoradiotherapy in glioblastoma. J Clin Oncol. 2008;26(18):3015-24.

19. Shai $R$, et al. Gene expression profiling identifies molecular subtypes of gliomas. Oncogene. 2003;22(31):4918-23.

20. Sun $L$, et al. Neuronal and glioma-derived stem cell factor induces angiogenesis within the brain. Cancer Cell. 2006;9(4):287-300.

21. Vermes I, et al. A novel assay for apoptosis. Flow cytometric detection of phosphatidylserine expression on early apoptotic cells using fluorescein labelled Annexin V. J Immunol Methods. 1995;184(1):39-51.

22. Favaloro B, et al. Role of apoptosis in disease. Aging (Albany NY). 2012;4(5): $330-49$.

23. Kenmochi N, et al. A map of 75 human ribosomal protein genes. Genome Res. 1998;8(5):509-23.

24. Zhao X, et al. Decreased expression of RPS15A suppresses proliferation of lung cancer cells. Tumour Biol. 2015;36(9):6733-40.

25. Li P, et al. Cytochrome $\mathrm{c}$ and dATP-dependent formation of Apaf-1/caspase9 complex initiates an apoptotic protease cascade. Cell. 1997;91(4):479-89.

26. Swanton $\mathrm{E}$, et al. $\mathrm{Bcl}-2$ regulates a caspase-3/caspase-2 apoptotic cascade in cytosolic extracts. Oncogene. 1999;18(10):1781-7.

27. Perry DK, et al. Bcl-2 acts upstream of the PARP protease and prevents its activation. Cell Death Differ. 1997;4(1):29-33.

28. Veenman L, Gavish M, Kugler W. Apoptosis induction by erucylphosphohomocholine via the $18 \mathrm{kDa}$ mitochondrial translocator protein: implications for cancer treatment. Anticancer Agents Med Chem. 2014:14(4):559-77.

Submit your next manuscript to BioMed Central and we will help you at every step:

- We accept pre-submission inquiries

- Our selector tool helps you to find the most relevant journal

- We provide round the clock customer support

- Convenient online submission

- Thorough peer review

- Inclusion in PubMed and all major indexing services

- Maximum visibility for your research

Submit your manuscript at www.biomedcentral.com/submit
) Biomed Central 